\title{
Prevalence of Emotions and Basic Feelings in Higher Education, Case: ESPOCH Marketing Career
}

\section{Prevalencia de Las Emociones y Sentimientos Básicos en la Educación Superior, Caso: Car- rera de Mercadotecnia ESPOCH}

\author{
W. Pilco and L. Vallejo
}

VII International Congress of

Science, Technology,

Entrepreneurship and

Innovation (SECTEI 2020)

Corresponding Author:

W. Pilco

luz.vallejo@espoch.edu.ec

Published: 26 August 2021

Production and Hosting by

Knowledge E

(c) W. Pilco and L. Vallejo. This article is distributed under the terms of the Creative

Commons Attribution

License, which permits unrestricted use and redistribution provided that the original author and source are credited.

Escuela de Ingeniería en Marketing, Facultad de Administración de Empresas, Escuela Superior Politécnica de Chimborazo, Riobamba, Ecuador

\section{Abstract}

This research aimed to determine the prevalence of basic emotions and feelings in higher education students, through a case study of students pursuing the marketing degree of the Polytechnic School of Chimborazo (ESPOCH). The research employed the MATEA model (fear, affection, sadness, anger and joy) and qualitative and quantitative approaches. The research design was descriptive, cross-sectional and non-experimental, and the methods used were inductive, deductive, analytical, synthetic and systemic. Data were collected through documentation and field review. The results indicated that there was a high level of fear, anger and joy in the students, a neutral level of sadness, and a positive feeling of affection. Given that anger and fear were identified at high levels in the students, we conclude that these feelings should be treated by ESPOCH's Department of Well-being, Polytechnic and Psychology.

Keywords: MATEA model, emotions, feelings, emotional codes.

\section{Resumen}

La investigación tiene como objetivo determinar la prevalencia de las emociones y sentimientos básicos en los estudiantes de educación superior, caso: Carrera de Mercadotecnia de la Escuela Superior Politécnica de Chimborazo (ESPOCH), se fundamenta en el modelo MATEA (miedo, afecto, tristeza, enojo y alegría) tiene un enfoque cualitativo y cuantitativo, de nivel descriptivo, de diseño transversal, no experimental, los métodos utilizados fueron inductivo, deductivo, analítico, sintético y sistémico en el proceso de revisión documental y de campo desarrollados en la presentación de resultados, discusión y conclusiones. Los resultados determinaron el sentimiento miedo su nivel es alto; el sentimiento afecto es positivo, el sentimiento tristeza se encuentra en nivel neutro, el sentimiento enojo su nivel es alto, el sentimiento alegría fue alto. Se concluye que los sentimientos enojo y miedo fueron identificados altos en los estudiantes, se recomienda que estos sentimientos deben ser tratados por los departamentos Bienestar Estudiantil Politécnico y Psicología de la ESPOCH.

Palabras Clave: modelo MATEA, emociones, sentimientos, códigos emocionales. 


\section{Introducción}

La prevalencia de las emociones y sentimientos básico en los estudiantes, se fundamenta en el análisis del modelo de la doctora Myriam Muñoz Polít; que a través de un acróstico 'MATEA' (Miedo, Afecto, Tristeza, Enojo, Alegría) asocia las emociones. EI acróstico forma la palabra AMATE que aplicado en forma literaria constituye una parte esencial en la experiencia humana.

A través de este modelo se analiza los siguientes sentimientos: el miedo que constituye una defensa o protección hacía alguna situación; la alegría que vivífica a la persona; la tristeza provoca un retiro hacia sí mismo; el enojo es un mecanismo de defensa hacía algo o alguien que ha traspasado el espacio vital en la persona. Cuando las emociones son controladas evitan conflictos interiores y exteriores, una de las formas de manejarlas cuando se relacionan a recuerdos de tristeza o de nostalgia es compartir los sentimientos y pensamientos con otros seres humanos cercanos para recibir consejo y motivación.

Cuando existe una excesiva tristeza se convierte en depresión, la furia o enojo en rencor, los temores o aprensiones en barreras que frenan el desarrollo personal y profesional de los seres humanos. Al identificar los sentimientos básicos con un modelo existente de aplicación didáctica permite tener una contextualización clara del tipo de personalidad del estudiante que será fácilmente identificado por el docente en el periodo académico de trabajo. La tendencia actual de la educación es formar al estudiante en valores integrales que son parte de la formación y no solo enseñar asignaturas de una malla de currícula sin saber si los estudiantes se encuentran bien emocionalmente, los docentes de la educación superior y de todos los niveles educativos deben trabajar en esta tendencia de formar y educar al mismo tiempo. Para formar y educar es importante identificar la prevalencia de las emociones y los sentimientos en los estudiantes. Se espera que los resultados de investigación generen un aporte educativo para el acompañamiento de tutorías y la toma de acciones para el departamento de Bienestar Estudiantil de las Universidades.

Considerando que el éxito de un estudiante no solo radica en la acumulación y aplicación de teorías de las distintas áreas del conocimiento, su parte emocional y sentimental también influyen en su formación académica, para obtener profesionales motivados y con aspiraciones de crecimiento y desarrollo personal en beneficio propio y de los demás.

En estudios recientes [1] demuestran que 'el aprendizaje lleva consigo temores, conflictos, ansiedades, del conocimiento anterior frente al nuevo'. Según [2] en su teoría del pensamiento manifiesta que: 'el aprendizaje genera experiencias emocionales frustrantes'. Pero no necesariamente son todas experiencias frustrantes, también existen experiencias cristalizantes que denotan alegría, afectividad, entusiasmo y tenacidad 
por seguir avanzando en las metas y objetivos que conlleva el estudio de una carrera universitaria.

Pero quien es el responsable de identificar la prevalencia de las emociones y sentimientos, el responsable directo en el aula de clase es el docente; a través de sus funciones de formador, es receptivo, juicioso y analítico, que saber escuchar, no solo oír la comunicación verbal y no verbal en el estudiante sin rebasar los espacios permitidos por el estudiante [3,4]. Manifiesta la importancia de utilizar de forma adecuada los códigos emocionales de motivación, tenacidad, de éxito personal y profesional, de alimentación y del buen vivir en los estudiantes.

Según $[1,5]$ coinciden que la formación es la acción educativa donde se producen nuevas capacidades para que los estudiantes puedan enfrentar situaciones relacionadas a su campo profesional con eficacia, efectividad y eficiencia; las decisiones que se tomen serán el fiel reflejo de una formación integral, no solo una enseñanza centrada en conocimientos técnicos científicos que no valore las emociones (reacciones inmediatas del cuerpo frente a un determinado suceso o acontecimiento del diario vivir) y sentimientos (estados de ánimo que expresa una persona como resultado de una emoción) de los estudiantes.

El objetivo de la investigación es determinar la prevalencia de las emociones y sentimientos con la aplicación del modelo MATEA, en los estudiantes de la Escuela de Marketing de la Facultad de Administración de Empresas de la Escuela Superior Politécnica de Chimborazo. Con el fin de identificar las emociones y sentimientos que prevalecen en los estudiantes para brindar un seguimiento y acompañamiento de docentes, tutores y los departamentos de bienestar estudiantil Politécnico y de Psicología de la ESPOCH.

\subsection{Modelo MATEA}

Los docentes al formar y educar en el aula de clases en las capacidades 'el saber y el saber hacer' de la misma manera es importante que el docente identifique la prevalencia de las emociones y sentimientos de sus estudiantes, esto se consigue en el ejercitarse en el sentir y el ser, de quienes están receptando la información; de esta manera ayudamos a nuestros estudiantes a esforzarse y mejoramos una sociedad emocionalmente estable. Para identificar la prevalencia de las emociones y sentimientos el docente puede aplicar técnicas cualitativas de investigación como entrevistas a profundidad, la observación participativa o talleres didácticos, test o el modelo MATEA.

El modelo MATEA de la doctora Miriam Muñoz es una metodología emocional que permite identificar que sentimientos básicos que un estudiante proyecta a través de su comportamiento, la pregunta es ¿cuáles son aquellos comportamientos que fácilmente 
observa en los estudiantes universitarios? Es así, que los docenes rompen el paradigma 'que el aula de clase no es solo un espacio de enseñanza' sino deben convertirse en espacios de formación integral.

El método MATEA explica las emociones y sentimientos:

1. Miedo: Es una reacción a lo nuevo o desconocido, este miedo surge al momento de experimentar un examen, una nueva tarea, un trabajo académico o una nueva responsabilidad, al no contestar bien una pregunta en la clase, al no haber estudiado el tema del examen, al recibir una nota, a no aprobar la materia, y como continuar son sus estudios. En el aula de clase es muy común este sentimiento, que de manera constante el estudiante se enfrenta a nuevas decisiones que determinarán el rumbo de su carrera profesional.

2. Afecto: Es la necesidad de crear amistades dentro del entorno educativo, es el sentido de pertenencia al grupo, con sus compañeros dentro del aula de clases. En el afecto la personalidad y liderazgo consiste en la aceptación del grupo, de sus compañeros. En sentido negativo este sentimiento debe ser tomado en cuenta debido a que una mala decisión, puede conllevar a críticas en el grupo.

3. Tristeza: Es el sentimiento cuando se falla en algún objetivo, la vida estudiantil está compuesta por retos constantes, si el estudiante no está capacitado para salir de este sentimiento puede perjudicar de manera directa su vida estudiantil y profesional. En sentido positivo sirve de incentivo para mejorar el entorno.

4. Enojo: Es el más común en la vida estudiantil, es una reacción al sentido de frustración. Puede desatarse por diversos estímulos desde: una carrera indeseada y obligada, una mala relación con compañeros y docentes, un mal desenvolvimiento social, una mala capacitación, una competencia de poderes, saberes y conocimientos. El enojo está presente en muchas ocasiones, y si no sabemos manejarlo adecuadamente, puede afectar física y mentalmente.

5. Alegría: Es un sentimiento o emoción que expresamos con una sonrisa y con energía. Se expresa cuando nos sentimos muy felices por algo que logramos como: un buen examen, compartir con los compañeros de clases, el aprendizaje o incluso sentir alegría por algo que se hizo bien o estar con alguien que queremos. Es un sentimiento bueno y positivo que se encamina a la acción.

El mal manejo de emociones puede perjudicar nuestra la vida estudiantil, académicamente y profesionalmente de manera significativa, aquí es donde entra la inteligencia emocional del estudiante y la capacidad de renovarse de manera constante, los estímulos provocan emociones y sentimiento, pero no siempre estos afecta del mismo modo a cada estudiante es decir 'Las emociones y sentimiento depende de la personalidad 
del estudiante y su punto de control interno o externo para que le afecte o no' así la frase de uso común 'lo que me afecta a mí no necesariamente puede afectar al otro. El manejo de estos sentimientos tiene mucho que ver con los filtros de percepción y control de cada persona'.

El modelo MATEA es un acróstico que representa las emociones y sentimientos básicos como: Miedo, Afecto, Tristeza, Enojo, Alegría; se aplica una escala de Likert del 1 al 5 para definir las emociones más recurrentes, para luego transformar a fracciones aritméticas y un análisis descriptivo.

Las emociones se refieren al dominio de acciones en el cual el ser humano se mueve. Así [6] define 'las emociones son disposiciones corporales dinámicas que especifican el dominio de acciones'. Por lo tanto, no hay acción humana sin una acción, que se fundamente en un estímulo recibido interno o externo.

Por ejemplo, si un estudiante se encuentra bajo la emoción del miedo, en una situación académica que a él le atemoriza, como un examen, es posible que tiemble la voz, su pulso se incremente, se encuentra agitado y actúe diferente con sus compañeros de clase. Un estudiante al exponer una temática al encontrarse frente a sus compañeros puede tener miedo de realizar su exposición, así sus manos sudan y su corazón late más rápido. Más aun si el docente es de matemáticas y es la materia que posiblemente no le guste al estudiante, puede pasar al frente a realizar un ejercicio o dar la lección mientras se dice internamente: 'voy a obtener cero en la clase de hoy' o más aún cuando el docente dice frases como: 'de este paralelo solo pasarán 5 estudiantes' el miedo en el estudiante se intensifica.

Otro ejemplo en los estudiantes, es cuando existe una baja calificación en una evaluación, esto genera emociones como pueden ser tristeza, ira consigo mismo o con el docente, desmotivación o motivación esto dependerá de la personalidad y el autocontrol del estudiante.

El neurobiólogo Antonio Damásio (2005) ‘En busca de Spinoza’ destaca el papel de las emociones en el pensamiento humano, su análisis integra evidencia neurocientífica fundamentada en el filósofo holandés Spinoza (1632-1677) quien consideraba a los sentimientos y emociones como aspectos centrales de la condición humana. A continuación, se detalla algunos conceptos de Damásio:

1. Una emoción como la felicidad, tristeza, vergüenza o simpatía es un conjunto complejo de respuestas químicas y neuronales que forman un patrón distintivo.

2. Las respuestas son producidas por el cerebro cuando detecta un estímulo que es el objeto cuya presencia y re-memorización metal, desencadena una emoción, estas respuestas son automáticas.

3. El cerebro está preparado para responder con repertorios específicos de acción en el momento y de otros aprendidos por la experiencia. 
4. El resultado inmediato de estas respuestas directa o indirectamente es un cambio temporal en el estado del propio cuerpo y en el estado de las estructuras cerebrales que cartografían el cuerpo y sostiene el pensamiento.

5. El resultado último de las respuestas directas o indirectas es el actuar al organismo en circunstancias propicias para la supervivencia y el bienestar. [6]

Las emociones modifican el estado del cuerpo de una mamera que puede o no manifestarse a simple vista, son automáticas, aunque en ciertos casos modulares y no necesariamente existe conciencia plena de sus consecuencias cuando se disparan. Desde el punto de vista biológico las emociones están en el organismo para su bienestar y supervivencia.

Las emociones preceden a los sentimientos tanto en el momento de experimentar una emoción como evolutivamente. En la evolución los mecanismos cerebrales que sustentan las respuestas emocionales se forman antes de los sentimientos. Las emociones son funcionales en el sistema de regulación vital, están dirigidas a evitar peligros, ayudar al organismo a sacar partido de una oportunidad o facilitar las relaciones sociales. Las emociones en la vida cotidiana contribuyen a la sobrevivencia [7].

Según Damásio la evolución humana ensamblo la maquina cerebral de la emoción y el sentimiento en etapas: Primero se producen las reacciones a objetos y circunstancias, luego de desarrolló los mecanismos para producir un mapa cerebral y después una representación mental del estado resultante del organismo: Los sentimientos. Las emociones permiten responder de manera efectiva pero no creativa a las circunstancias favorables o amenazas para la supervivencia. Los sentimientos introdujeron un alerta metal y potenciaron su impacto de las emociones al afectar de manera permanente la atención y la memoria. Así juntamente con los recuerdos, la imaginación y el razonamiento, los sentimientos posibilitaron la producción de respuestas nuevas, no estereotipadas. Entonces en el principio fue la emoción que en el organismo produce una acción y este genera el sentimiento.

Los sentimientos y las emociones son fundamentales en las relaciones sociales y en la toma de decisiones que requiere la vida social, es decir en el razonamiento. Las emociones positivas o negativas y los sentimientos que e ellas siguen, están directamente implicados en nuestras experiencias sociales. Diferentes opciones de acción y diferentes resultados futuros están asociados a diferentes emociones/sentimientos, que, en una situación dada, posibilitan el proceso de toma de decisiones de manera autónoma y apropiada. Las señales emocionales no son sustitutos del razonamiento adecuado o un comportamiento razonables en ciertas circunstancias. Una señal emocional debe operar enteramente bajo el control de la conciencia, afecta la atención y la memoria de trabajo, de tal manera que el proceso de toma decisiones resulta sesgado sin darnos cuenta. Así la sabiduría popular afirma frases como 'Mi corazón me decía que 
debía hacer' o 'sentía que no debía hacer'. Es importante remarcar que las emociones son intrínsecamente emocionales, en el sentido que conducen a la mejor solución en términos de supervivencia [8].

Mientras vives a través de los días y años, estás continuamente experimentando emociones de algún tipo u otro. La vida puede ser difícil y las emociones a veces pueden ser abrumadoras. Todos nosotros experimentamos extremos emocionales positivos y negativos por momentos. Cuando son negativos producen enojo, o un período temporal de pena o depresión, esta energía emocional negativa puede permanecer dentro de tu cuerpo causando un significativo estrés físico y emocional [9].

Entonces que hacen los estímulos, afirma [10], 'los estímulos emocionales interactúan en las habilidades cognitivas, son palabras, frases o gestos conscientes o inconscientes que se manifiestan en el proceso enseñanza- aprendizaje y se convierten en sentimientos'.

Según el Dr. Brandley Nelson manifiesta que las emociones atrapadas (sentimientos) pueden hacer que se forme suposiciones incorrectas, reacciones de manera exagerada frente a comentarios inocentes, se malinterprete una conducta y cause un cortocircuito en las relaciones. Aún peor, las emociones pueden crear depresión, ansiedad y otros sentimientos indeseables que pareciera que no se pueden resolver. Pueden interferir en el funcionamiento correcto de los órganos y tejidos del cuerpo, causando estragos en la salud física, causando dolor, fatiga y enfermedad. Sin embargo, no importa cuán grande sea el sufrimiento, la energía invisible de las emociones atrapadas quedará sin ser diagnosticada por la medicina convencional, aun siendo un factor de causa mayor en las dificultades físicas y emocionales [11]

Las emociones y sentimientos que se generan en el aula de clase son el resultado de la interacción en el proceso de enseñanza aprendizaje o interaprendizaje de las diferentes culturas, tanto del docente como del estudiante que se expresan de manera proactiva, reactiva y retroactiva [12].

Estudios realizados por [13] señalan la existencia de estados afectivos positivos o negativos contribuyen u obstaculizan los procesos de enseñanza-aprendizaje, factores que se deben tomar en cuenta en dicho proceso. Así mismo [14] señalan que es fundamental comprender la apreciación de los estudiantes frente al proceso de aprendizaje, en función del grado de desafío y familiaridad que representa para ellos lograr sus metas individuales y comprender las emociones que sienten en estas situaciones ( $p$. 449).

Por su parte, [15] identifican que los estudiantes se percatan de una variedad de emociones relacionadas con el proceso de aprendizaje en la educación, demuestra que las emociones positivas como el placer de aprender, la esperanza, el orgullo, la admiración o la empatía están presentes al igual que las emociones negativas, 
concluyendo el estudio: las emociones están interrelacionadas con componentes esenciales del aprendizaje (p. 91).

En su estudio, [16] identifican una presencia significativa de emociones positivas en relación a las negativas en el proceso de aprendizaje online a diferencia del presencial, demuestra emociones positivas que experimentan los estudiantes durante las actividades de aprendizaje en entorno virtual son el alivio y la alegría, mientras que las emociones negativas experimentadas son preocupación-tensión y confusión desorientación.

Pekrun et al. (2010) identifican dos tipos de emociones en relación con el rendimiento académico; las emociones de actividad, que son actividades de aprendizaje en sí mismas y las emociones de resultados, que son las que resultan como consecuencia de los resultados obtenidos en esas actividades, identificando la limitada atención que se ha presentado a las emociones relacionadas con las actividades de aprendizaje tales como el aburrimiento, la indiferencia o el entusiasmo, en contraste con el interés provocado por las emociones relacionadas a los resultados como el orgullo, la ansiedad o la vergüenza (p. 531).

Por su parte [17] quienes estudian los estados afectivos de los discentes en relación con varias actividades educativas, identificando que los estudiantes experimentan estados afectivos que indican ansiedad (miedo, preocupación, estrés, angustia) al igual que los estados afectivos que indican motivación (entusiasmo, orgullo, bienestar, etc.) y la relación de estados afectivos contribuye al proceso de aprendizaje (p. 195)

Algunos estudios de [18] confirman la presencia de ansiedad en determinadas situaciones de aprendizaje, puede estar asociadas a factores como la asunción de un nuevo rol del educando y el reto que un conocimiento nuevo supone sobre la estructura de un conocimiento previo del estudiante, el desconocimiento sobre las propias capacidades para enfrentar dichos retos y la evocación de experiencias negativas previas de aprendizaje (p. 435).

Así [13] manifiestan que la ansiedad o tensión en relación con el conocimiento ya estructurado razón para que el docente despierte el deseo de aprender, generar curiosidad y mantener la atención focalizada.

Según Damásio las emociones se clasifican en tres categorías: Emociones de fondo, primarias y sociales:

Emociones de fondo: No son especialmente visibles Enel comportamiento, son expresiones compuestas de acciones reguladoras que se intersecan en cada momento de cotidiano de la vida, se denominan estados de ánimo, bueno, malo o intermedio.

Emociones primarias o básicas: Son propias del ser humano y son las que incluimos en determinados momentos o situaciones como: Ira, asco, sorpresa, miedo, la tristeza y la felicidad. 
Table 1

Diferencias entre emoción y sentimiento.

\section{Emociones}

Las emociones son estados físicos que surgen como respuesta a estímulos externos

Preceden el sentimiento

Pueden ser fácilmente observables por otros

Aparecen de forma brusca y son intensos

Son estados afectivos pasajeros

Son olvidadas fácilmente, es espontanea de corta duración

Pertenece al cuerpo

Elaborado por: Pilco, W. \& Vallejo L.

\section{Sentimientos}

Los sentimientos son asociaciones mentales y reacciones a las emociones

Posterior a la emoción

No son fáciles de observar por otros

Se presentan con moderada intensidad y no bruscamente

Son estados afectivos de carácter duradero

Son resultados de vivencias y experiencias que dejan huella

Pertenece a la mente

Table 2

Emociones, reacción, manejo positivo y manejo Patológico.

Emoción
Miedo
Alegría
Tristeza
Enojo
Afecto

Reacción
Autoprotección,
protección
Energía, vitalidad, fuerza,
voluntad, reanimar y
confortarse
Introspección, meditar y
reflexión
Defensa, limites
Vinculación ladaptación

Elaborado por: Pilco, W. \& Vallejo L.

Manejo positivo
Seguridad, fortaleza,
precaución
Gozo, disfrute, optimismo
Autoconocimiento,
profundidad
Seguridad, confianza y
firmeza
Autoestima y estima al
otro

Manejo patológico
Ansiedad, pánico, fobias
Euforia, manía, caprichos,
furor
Melancolía, nostalgia
asilamiento, depresión
Ira, rencor, apatía, odio,
resentimiento
Dependencia y
codependencia

Emociones sociales: Incluyen la simpatía, turbación, desdén, admiración, gratitud, indignación, celos, culpa, entre otras, que un complejo entramado de respuestas reguladoras. Estas respuestas sociales están presentes en humanos y en especias animales mamíferos como el Chimpancés, los delfines y lobos, en las especies de insectos como las abejas y las hormigas. Para el presente estudio se trabajan con las emociones primarias y básicas que se relaciona con el modelo MATEA.

El modelo MATEA considera las cinco emociones básicas que son reacciones primitivas que el ser humano comparte con los mamíferos, con la diferencia de los humanos somos conscientes de ellas al sentirlas. Tales emociones permiten al organismo cubrir objetivos de supervivencia, es así que no se siente como dice la frase 'a lo tonto' o de forma exagera. La emoción el miedo el objetivo es la protección, el afecto el objetivo es la vinculación y adaptación, la Tristeza el objetivo es el retiro o aislamiento cuando 
sentimos tristeza nuestro organismo nos está diciendo 'retírate de ahí y regresa a estar contigo mismo', el enojo el objetivo es la defensa, la alegría el objetivo es la vivificar, es la fuerza interna y la energía, es la batería de la existencia que fomenta el trabajo y la acción con voluntad.

\section{Materiales y Métodos}

El estudio de las prevalencias de las emociones y sentimientos se aplicó a una población de 430 estudiantes de la carreta de Mercadotecnia de la Facultad de Administración de Empresas (FADE) de la Escuela Superior Politécnica de Chimborazo (ESPOCH). La población de estudiantes de primero a décimo semestre en total de 438 estudiantes legalmente matriculados, la muestra fue 102 estudiantes en un muestreo no probabilístico.

El estudio realizado tiene un enfoque cualitativo cuantitativo que a través de entrevistas se determinaron las variables de estudio, y usando un cuestionario estructurado no disfrazado se desarrolló el trabajo de campo para que los datos se conviertan en información. El estudio se fundamenta en el modelo MATEA (miedo, afecto, tristeza, enojo y alegría), el nivel de la investigación es descriptivo, se pretende presentar el contexto con detalles porcentuales de las emociones básicas.

El diseño es no experimental y transversal, debido a que se toman los datos tal y como se encuentran en una realidad inmediata, es decir a los estudiantes en las aulas de clases, por una sola vez (una medición). El cuestionario es válido por dos criterios cualitativos: Criterio de validez de contenido; las variables emocionales reflejan los objetivos de la investigación, además de indicar, que se está usando un modelo teórico con fines descriptivos: MATEA (Muñoz, M., Castro, M., 2016). El otro criterio es la validación de constructo, es decir, se está midiendo de forma descriptiva el comportamiento de las emociones que predominan en los estudiantes. No es posible usar un criterio cuantitativo (Alfa de Cron Bach), por cuanto las varianzas generan valores negativos en su consistencia interna. Los métodos utilizados en la investigación fueron el inductivo, deductivo, analítico, sintético y sistémico en el proceso de revisión documental, de campo y la presentación de resultados, la discusión y conclusiones. El procedimiento fue el siguiente:

1. Se enlista en una hoja de papel el acróstico:
(a) Miedo
(b) Afecto
(c) Tristeza
(d) Enojo 


\section{(e) Alegría}

2. Se solicita al estudiante que identifique con el número 1 el sentimiento más conocido y fácil de reconocer.

3. Se pide colocar el número 5 el sentimiento más difícil de reconocer y/o contactar.

4. Se pide que ubique el número 2 el sentimiento que después del número 1 sea fácil de identificar.

5. Seguido que ponga el número 4 en el sentimiento que después del número 5 se más difícil de reconocer.

6. El número 3 va en el sentimiento considerado neutro que tiene una proporción equilibrada, ni en más ni en menos en el comportamiento habitual.

7. Análisis interpretativo:

(a) El sentimiento marcado con el número 1 cubre al 5;

(b) El sentimiento marcado con el número 2 cubre al 4;

(c) El sentimiento marcado con el número 3 es aquel que se presenta en una justa proporción de equilibrio o neutro:

Esta representación fraccionaria se la aplica en un análisis individual en cada estudiante para realizar un análisis interpretativo. Normalmente las personas suelen exagerar los sentimientos, si el sentimiento es más intenso se coloca 1 y 2 para disminuir o tapar los sentimientos se coloca 4 y 5 estos sentimientos son más difíciles de contactar con ellos. El análisis deriva del número 4 y 5 son los que más hay que trabajar ya que las necesidades que están en ahí en estos números no están bien satisfechas.

Así, si son altos en el miedo significa que no sabemos protegernos bien, si es alto el afecto, que tenemos problemas con la vinculación y adaptación, si es alto en tristeza que nos cuesta trabajo estar a solas conmigo y me quedo pegado al mundo se necesita introspección y reflexión, si es alto en enojo tengo dificultad para ponerles límites al mundo y si es alto la alegría viene a significar que hay algún problema de la fuerza y la energía de mi vitalidad y es posible que me cueste trabajo la vida.

Los resultados de la investigación permitirán dar seguimiento y acompañamiento a los estudiantes a través de los departamentos de Bienestar Estudiantil Médico y Psicológico de la ESPOCH.

\section{Resultados y Discusión}

La prevalencia de las emociones y sentimientos aplicando el modelo MATEA, en una muestra de 102 estudiantes de la carrera de Mercadotecnia de la FADE-ESPOCH, 
institución pública de educación superior que tienen como principal característica demográfica el 60\% de estudiantes provienen de otras ciudades y regiones (costa, sierra y oriente) a la ciudad de Riobamba provincia de Chimborazo.

A través del muestreo no probabilístico se identificó en las distintas aulas de clase y paralelos a 51 estudiantes mujeres y 51 estudiantes hombres para que realicen la encuesta con la metodología de MATEA para identificar la prevalencia de las emociones y sentimientos en los estudiantes. Los principales resultados estadísticos encontrados una vez realizado el trabajo de campo con la intervención de los compañeros estudiantes del 5to nivel paralelo 1 en la promoción académica septiembre 2019-febrero 2020 fueron:

Table 3

Género de los estudiantes de la Escuela de Mercadotecnia.

\begin{tabular}{|c|c|c|c|c|c|}
\hline \multicolumn{6}{|l|}{ Género } \\
\hline & & Frecuencia & Porcentaje & $\begin{array}{l}\text { Porcentaje } \\
\text { válido }\end{array}$ & $\begin{array}{l}\text { Porcentaje } \\
\text { acumulado }\end{array}$ \\
\hline \multirow[t]{3}{*}{ Válido } & Femenino & 51 & 50,0 & 50,0 & 50,0 \\
\hline & Masculino & 51 & 50,0 & 50,0 & 1000 \\
\hline & Total & 102 & 100,0 & 100,0 & \\
\hline
\end{tabular}

Elaborado por: Pilco, W. \& Vallejo L.

La Tabla 3 muestra el género de los estudiantes, dividido en forma equitativa el 50\% al género femenino y el otro $50 \%$ al género masculino, con la intención de realizar tabulaciones cruzadas.

Los resultados descriptivos generales son:

\section{Table 4}

Sentimiento miedo.

\begin{tabular}{|c|c|c|c|c|}
\hline \multicolumn{2}{|l|}{ MIEDO } & \multirow{2}{*}{$\begin{array}{l}\text { Frecue ncia } \\
8\end{array}$} & \multirow{2}{*}{$\begin{array}{l}\text { Por cen taj e } \\
7,8\end{array}$} & \multirow{2}{*}{$\begin{array}{l}\text { Frecuen cie } \\
\text { acumul ada } \\
7,8\end{array}$} \\
\hline Válido & $\begin{array}{l}\text { Sentimiento más conocido y } \\
\text { fácil de reconocer }\end{array}$ & & & \\
\hline & $\begin{array}{l}\text { Sentimiento que después del } 1 \\
\text { es más fácil de reconocer o } \\
\text { identificar }\end{array}$ & 15 & 14,7 & 22,5 \\
\hline & Sentimiento neutro & 25 & 24,5 & 47,1 \\
\hline & $\begin{array}{l}\text { Sentimiento que después del } 5 \\
\text { es difícil de reconocer }\end{array}$ & 27 & 26,5 & 73,5 \\
\hline & Sentimiento difícil de reconocer & 27 & 26,5 & 100,0 \\
\hline & Total & 102 & 100,0 & \\
\hline
\end{tabular}

Elaborado por: Pilco, W. \& Vallejo L. 
La Tabla 4 muestra que el sentimiento miedo es el sentimiento difícil de reconocer, pero está presente en los estudiantes de la Escuela de Mercadotecnia, en las actividades académicas como: Exposiciones, trabajos de defensa y actividades que desconocen cómo se deben realizar, significa que el estudiante necesita sentirse protegido para actuar y esto se fundamenta en dominar el conocimiento y estar seguro que si pueden desarrollar sus actividades sin temor.

\section{Table 5}

Sentimiento afecto.

\begin{tabular}{|c|c|c|c|c|}
\hline \multicolumn{2}{|c|}{ AFECTO } & \multirow{2}{*}{$\begin{array}{l}\text { Fre cue nci a } \\
14\end{array}$} & \multirow{2}{*}{$\begin{array}{l}\text { Porce ntaje } \\
13,7\end{array}$} & \multirow{2}{*}{$\begin{array}{l}\text { Porcent aje } \\
\text { acumul ado } \\
13,7\end{array}$} \\
\hline Válido & $\begin{array}{l}\text { Sentimiento más conocido y } \\
\text { fácil de reconocer }\end{array}$ & & & \\
\hline & $\begin{array}{l}\text { Sentimiento que después del } 1 \\
\text { es más fácil de reconocer o } \\
\text { identificar }\end{array}$ & 27 & 26,5 & 40,2 \\
\hline & Sentimiento neutro & 19 & 18,6 & 58,8 \\
\hline & $\begin{array}{l}\text { Sentimiento que después del } 5 \\
\text { es difícil de reconocer }\end{array}$ & 19 & 18,6 & 77,5 \\
\hline & Sentimiento difícil de reconocer & 23 & 22,5 & 100,0 \\
\hline & Total & 102 & 100,0 & \\
\hline
\end{tabular}

Elaborado por: Pilco, W. \& Vallejo L.

La Tabla 5 muestra el sentimiento afecto tiene un valor alto del 26,5\% es una expresión corporal habitual identificado en sentido positivo, demuestran que los estudiantes en sus actividades diarias educativas como: Al realizar sus tareas y la relación con sus docentes, compañeros de clase, y con la misma institución existe este sentimiento. En sentido negativo la falta de afecto; significa que los estudiantes tienen carencias en la vinculación y la adaptación.

La Tabla 6 muestra el sentimiento tristeza es un sentimiento neutro; es decir, que no tiene mayor significancia en los estudiantes de la Escuela de Mercadotecnia a pesar de su condición de encontrarse fuera de sus lugares de origen y su familia. Cuando este nivel es alto marca la pauta para el seguimiento emocional en los estudiantes; debido a que la excesiva tristeza se convierta en depresión y luego en una alerta emocional para ser tratado por especialistas del departamento de Bienestar Estudiantil Politécnico.

Los mapas relacionados a la tristeza corresponden a desequilibrios del organismo y reducen la facilidad de acción. El dolor, los síntomas de enfermedad indican un desequilibrio en las funciones vitales, si no se contrarrestan, la situación puede propiciar una enfermedad como la depresión que incluso puede ocasionar la muerte [7] (p. 134).

La Tabla 7 muestra el sentimiento enojo con el $27,5 \%$ un porcentaje alto en el 'sentimiento difícil de reconocer', así, el comportamiento del estudiante analizado de forma positiva refleja la defensa en el entorno y la creación de límites al realizar 
Table 6

Sentimiento tristeza.

\begin{tabular}{|c|c|c|c|c|}
\hline \multicolumn{2}{|c|}{ TRISTEZA } & \multirow{2}{*}{$\begin{array}{l}\text { Frecuencia } \\
12\end{array}$} & \multirow{2}{*}{$\begin{array}{l}\text { Porcentaje } \\
11,8\end{array}$} & \multirow{2}{*}{$\begin{array}{l}\text { Porcentaje } \\
\text { acumulado } \\
11,8\end{array}$} \\
\hline Válido & $\begin{array}{l}\text { Sentimiento más conocido y } \\
\text { fácil de reconocer }\end{array}$ & & & \\
\hline & $\begin{array}{l}\text { Sentimiento que después del } 1 \\
\text { es más fácil de reconocer o } \\
\text { identificar }\end{array}$ & 18 & 17,6 & 29,4 \\
\hline & Sentimiento neutro & 25 & 24,5 & 53,9 \\
\hline & $\begin{array}{l}\text { Sentimiento que después del } 5 \\
\text { es difícil de reconocer }\end{array}$ & 28 & 27,5 & 81,4 \\
\hline & Sentimiento difícil de reconocer & 19 & 18,6 & 100,0 \\
\hline & Total & 102 & 100,0 & \\
\hline
\end{tabular}

Elaborado por: Pilco, W. \& Vallejo L.

Table 7

Sentimiento enojo.

\begin{tabular}{ll|l} 
ENOJO & & Frecuencia \\
\hline Válido & $\begin{array}{l}\text { Sentimiento más conocido y } \\
\text { fácil de reconocer }\end{array}$ & 16 \\
$\begin{array}{l}\text { Sentimiento que después del } \\
\text { es más fácil de reconocer o } \\
\text { identificar }\end{array}$ & 19 \\
& $\begin{array}{l}\text { Sentimiento neutro } \\
\text { Sentimiento que después del } 5\end{array}$ & 22 \\
\hline $\begin{array}{l}\text { es difícil de reconocer } \\
\text { Sentimiento difícil de reconocer }\end{array}$ & 28 \\
\hline Total & 102 \\
\hline
\end{tabular}

\begin{tabular}{|l|l|}
\hline Porcentaje & $\begin{array}{l}\text { Porcentaje } \\
\text { acumulado } \\
15,7\end{array}$ \\
\hline 18,6 & 34,3 \\
\hline 16,7 & 51,0 \\
\hline 21,6 & 72,5 \\
\hline 27,5 & 100,0 \\
\hline 100,0 & \\
\hline
\end{tabular}

Elaborado por: Pilco, W. \& Vallejo L.

diariamente las actividades educativas, con sus compañeros y docentes; en sentido negativo, puede provocar rencor hacia otras personas, de ahí la importancia del docente en saber identificarlas y formar en las emociones y no solo enseñanza académica. En este sentimiento es importante la intervención y acompañamiento de los tutores académicos y el departamento de Bienestar estudiantil Politécnico.

La Tabla 8 muestra que el sentimiento más conocido y fácil de reconocer e, la Alegría con el $51 \%$ es decir, que existe una prevalencia de este sentimiento en los estudiantes de la carrera de Mercadotecnia, éste sentimiento básico se identifica con facilidad en momentos como: La alegría que aplican al aprender y adquirir nuevos conocimientos, al compartir experiencias con sus compañeros que se convierten en su segunda familia, más aún cuando la gran mayoría de estudiantes provienen de otras ciudades y se encuentran estudiando en la ciudad de Riobamba. 
Table 8

Sentimiento alegría.

\begin{tabular}{|c|c|c|c|c|}
\hline \multicolumn{2}{|c|}{ ALEGRÍA } & \multirow{2}{*}{$\begin{array}{l}\text { Frecuencia } \\
52\end{array}$} & \multirow{2}{*}{$\begin{array}{l}\text { Porcentaje } \\
51,0\end{array}$} & \multirow{2}{*}{$\begin{array}{l}\text { Porcentaje } \\
\text { acumulado } \\
51,0\end{array}$} \\
\hline Válido & $\begin{array}{l}\text { Sentimiento más conocido y } \\
\text { fácil de reconocer }\end{array}$ & & & \\
\hline & $\begin{array}{l}\text { Sentimiento que después del } 1 \\
\text { es más fácil de reconocer o } \\
\text { identificar }\end{array}$ & 23 & 22,5 & 73,5 \\
\hline & Sentimiento neutro & 16 & 15,7 & 89,2 \\
\hline & $\begin{array}{l}\text { Sentimiento que después del } 5 \\
\text { es difícil de reconocer }\end{array}$ & 6 & 5,9 & 95,1 \\
\hline & Sentimiento difícil de reconocer & 5 & 4,9 & 100,0 \\
\hline & Total & 102 & 100,0 & \\
\hline
\end{tabular}

Elaborado por: Pilco, W. \& Vallejo L.

Los sentimientos que se identificaron son: El enojo y miedo los mismos que deben ser tratados por docentes, tutores; y por los departamentos: Bienestar Politécnico y Psicología de la ESPOCH, de esta manera se logrará en la Escuela de Marketing un precedente para aplicar el modelo MATEA en otras escuelas, otras facultades y universidades con el fin de lograr una formación integral en los estudiantes.

Los mapas asociados a la alegría, significan estados de equilibrio para el organismo, que pueden realmente presentarse en la mente como si ocurriera, por el simple hecho de pensar en cosas positivas, los estados alegres además de ser propicios para la supervivencia tienen el añadido de bienestar, y permiten en los estudiantes mayor capacidad para actuar. [8]

A continuación, se representa en la estadística descriptiva en los estudiantes en el sentimiento alegría, se ha identificado este sentimiento por sexo, y se ha planteado la pregunta ¿quiénes son más alegres, las mujeres o los hombres en la escuela de marketing - FADE -ESPOCH?

La Tabla 9 demuestra estadísticamente que las mujeres de la escuela de Mercadotecnia reconocen fácilmente este sentimiento alegría, en relación a los hombres; ésta se reconoce en las actividades diarias como estudiantes y se considera que las mujeres son más expresivas, afectivas y emocionales que los hombres.

La Figura 1 muestra la aplicación de las encuestas a los estudiantes de quinto semestre periodo comprendido septiembre 2019-febrero 2020 de la Escuela de Marketing, de la Facultad de Administración de Empresas de la Escuela Superior Politécnica de Chimborazo. 
Table 9

Análisis cruzado del sexo en estudiantes en el sentimiento Alegría.

\begin{tabular}{|c|c|c|c|c|c|c|c|}
\hline \multicolumn{8}{|c|}{ Análisis Cruzado Sexo en Estudiantes en el Sentimiento Alegría } \\
\hline \multicolumn{8}{|c|}{ Sentimiento Alegría } \\
\hline & & $\begin{array}{l}\text { Sentimiento } \\
\text { más } \\
\text { conocido y } \\
\text { fácil de } \\
\text { reconocer }\end{array}$ & $\begin{array}{l}\text { Sentimient } \\
\text { o que } \\
\text { después } \\
\text { del } 1 \text { es más } \\
\text { reconocer o } \\
\text { identificar }\end{array}$ & $\begin{array}{l}\text { Sentimiento } \\
\text { neutro }\end{array}$ & $\begin{array}{l}\text { Sentimiento } \\
\text { que } \\
\text { después } \\
\text { del } 5 \text { es } \\
\text { difícil de } \\
\text { reconocer }\end{array}$ & $\begin{array}{l}\text { Sentimiento } \\
\text { difícil de } \\
\text { reconocer }\end{array}$ & Total \\
\hline Sexo en & Mujeres & 31 & 8 & 8 & 3 & 1 & 51 \\
\hline estudian tes & Hombres & 21 & 15 & 8 & 3 & 4 & 51 \\
\hline Total & & 52 & 23 & 16 & 6 & 5 & 102 \\
\hline
\end{tabular}

Elaborado por: Pilco, W. \& Vallejo L.
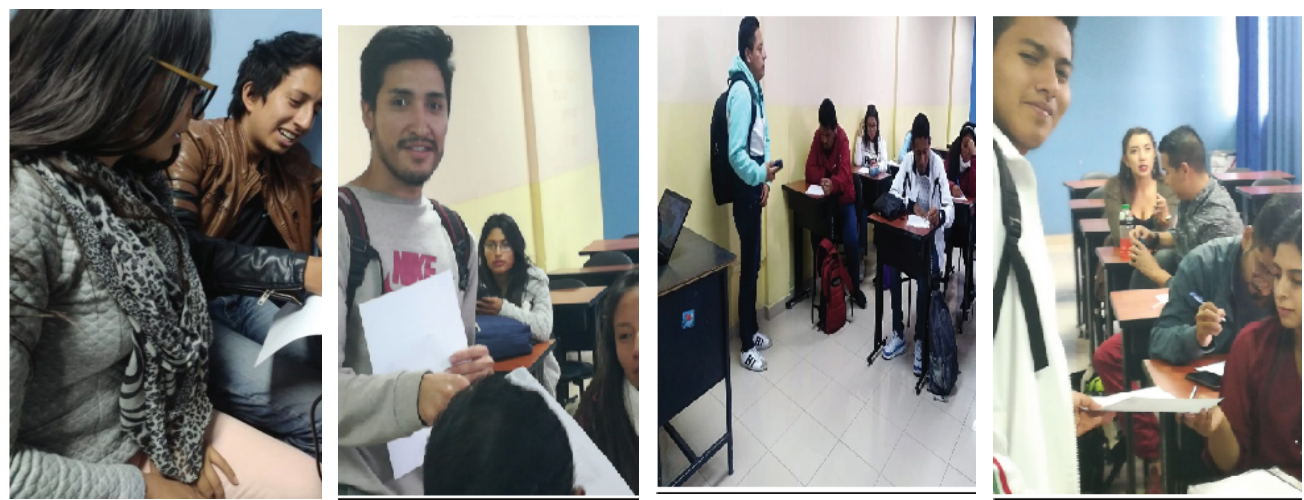

Figure 1

Collage de estudiantes aplicando la encuesta del modelo MATEA. Elaborado por: Pilco, W. \& Vallejo $L$.

Los sentimientos se convierten en sensores mentales en el interior del organismo que permiten el equilibrio interno en las personas. Es importante recalcar que la alegría y la tristeza permiten en los estudiantes maniobrar para situarse en estados de supervivencia óptima. Los sentimientos y emociones son fundamentales en las relaciones sociales y en la toma de 504

decisiones que se requiere la vida social; es decir, en el razonamiento. Las emociones positivas o negativas y los sentimientos que presiden están directamente implicados en nuestras experiencias sociales. Diferentes opciones de acción y resultados futuros en el estudiante están asociados a diferentes emociones y sentimientos, para que en una situación dada posibiliten el proceso de toma de decisiones de manera automática y apropiada.

Las emociones muchas veces son evidenciables en el lenguaje verbal (palabras como: no voy a poder estudiar, esta difícil la carrera, entre otras) que provocan malestar físico, desmotivación en los objetivos de vida, que en los estudiantes incide en que 
los estudiantes no saben que quieren en su vida futura, es así que [19]: manifiesta 'Las emociones no se limitan a lo que sentimos netamente en ese momento, sino que provoca una reacción en cadena en nuestro organismo y en nuestra conducta que son inmediatas'.

Para [20] la Educación Superior posibilita a los docentes una justificación para asumir la docencia como un estilo de vida, que permite reunir su propia perspectiva respecto a cómo ser, cómo actuar, cómo dimensionar su trabajo y asumir su estatus social educacional, con la concepción de formar y enseñar en una educación integral en el estudiante.

\section{Conclusiones}

El estudio de la prevalencia de las emociones y sentimientos se realizó mediante la aplicación del modelo MATEA en los estudiantes de la Escuela de Marketing de la Facultad de Administración de Empresas de la Escuela Superior Politécnica de Chimborazo, y se llegó a las siguientes conclusiones:

El método MATEA de fácil aplicación, permitió identificar las emociones y sentimientos en los estudiantes de la carrera de marketing, sus resultados determinaron que: El sentimiento miedo, es alto en los estudiantes, y no se identifica claramente según él método, esto se debe a que los estudiantes sienten miedo en el transcurso de las actividades académicas como: un tema nuevo, cuando no estudia, no entienden cómo deben realizar las actividades, al contestar, exponer o simplemente tiene miedo a preguntar o equivocarse. El sentimiento afecto, fue fácilmente identificado en los estudiantes, en sentido positivo tiene efecto en la vinculación y adaptación del estudiante, también se ve reflejado en el afecto a por sus compañeros, docentes y la institución.

El sentimiento tristeza, se encuentra en nivel neutro, significa que los estudiantes que se encuentran en este nivel no les afecta el estar solos, en el sentido de encontrarse fuera de sus hogares, al contrario, mantienen una buena relación social con sus compañeros, docentes y su entorno. El sentimiento enojo, fue difícil de reconocer en los estudiantes, su nivel fue alto, significa que los estudiantes tienen dificultad en poner límites en sus actividades y defenderse del entorno, el exceso de enojo puede afectar su rendimiento académico, y surge porque no saben decir 'no' en las decisiones diarias, en el sentido que se sobrecargan de trabajo cuando realizan actividades grupales y de esta manera afectan sus estados emocionales.

En el sentimiento alegría, fue muy fácil de detectar por parte de los estudiantes con un nivel alto, esto significa que la alegría, la fuerza de voluntad, la energía, el reanimarse y confortarse están presenten en los estudiantes de la escuela de mercadotecnia. Así se concluye que el enojo y el miedo se identificaron como altos y al ser de difícil de reconocer en los estudiantes éstos sentimientos deben ser tratados, es así que deben 
ser remitidos a los departamentos de Bienestar Estudiantil Politécnico y Psicología de la ESPOCH, por parte de los tutores mediante un informe de los resultados de la aplicación del modelo MATEA que permitieron identificar la prevalencia de estas emociones en los estudiantes, de uno u otro modo, afectan la salud emocional.

\section{Agradecimientos}

A los estudiantes de quinto semestre de la carrera de Mercadotecnia período comprendido octubre 2019-febrero 2020, muchas gracias por el entusiasmo aplicado al realizar esta actividad y en la aplicación de las encuestas, que facilitaron el presente trabajo de investigación.

\section{Conflicto de Intereses}

Los resultados de la aplicación del modelo MATEA, de una escuela a otra pueden variar, por este motivo cada caso será su resultado diferente; para posteriormente realizar un análisis interpretativo entre escuelas, estos resultados permitirán actuar a los departamentos indicados con el fin de realizar planes de capacitación y otras estrategias que faciliten generar un seguimiento respectivo en cada Escuela de manera individual.

\section{References}

[1] Sánchez L, Manrique M. El trabajo sobre las emociones en la formación de educadores en el nivel superior REXE. Revista de Estudios y Experiencias en Educación. 2018; 17(34).

[2] Castro M. Coaching en Acción. Herramientas de Intervención. $2^{\text {nd }}$ edition. Ciudad de México; 2016.

[3] Maturana HR. Cognição, ciência e vida cotidiana. Brasil: Editorial UFMG;2001.

[4] Sureda P. Emociones, sentimientos y razones en la Educación Matemática. Proyecto de Tesis Doctoral [Doctorado en Educación]. Facultad de Humanidades, UNCOR; 2006.

[5] Corica AR. Enseñanza de la Matemática en la Universidad. Emociones, sentimientos y Razonamientos [Doctorado en Educación]. Facultad de Humanidades, UNCOR;2005.

[6] Damásio A. El error de descartes. Ciudad Barcelona; 2001.

[7] Damásio A. En busca de Spinoza. Neurobiología de la emoción y los sentimientos. Ciudad Barcelona; 2005. 
[8] Otero M. Emociones, sentimientos y razonamientos en la didáctica de las ciencias. Revista Electrónica e Investigativa en Educación en Ciencias. 2006.

[9] Bradley N. El código de la emoción. Mesquite: Wellness Unmasked Publishing; 2007.

[10] Campos, AL. Neuroeducación: uniendo las neurociencias y la educación en la búsqueda del desarrollo humano. La educación, revista digital. 2010;143:14.

[11] Etchevers, N. Los nuevos códigos de la comunicación emocional utilizados en internet. Revista Electrónica Teoría de la Educación. Educación y Cultura en la Sociedad de la Información. 2006;7(2):92-106.

[12] Vallejo Luz, Pilco W, Guadalupe S, Silva L. Códigos emocionales en el proceso educativo en la educación superior. Caso ESPOCH. Congreso Internacional Redes INOVAESTIC; 2019; Alicante, España.

[13] Askham P. The feeling's mutual: Excitement, dread and trust in adult learning and teaching. Sheffield: Sheffield Hallam University; 2001.

[14] Wosnitza M, Volet S. Origin, direction and impact of emotions in social online learning. Learning and Instruction. 2005;15(5),449-64.

[15] Pekrun R, Goetz T, Daniels L, Stupniski R, Perry R. Boredom in achievement settings: Exploring control-value antecedents and performance outcomes of a neglected emotion. Journal of Educational Psychology. 2010;102(3):531-549.

[16] Rebollo M, García R, Barragán R, Buzón O, Vega L. Las emociones en el aprendizaje online. RELIEVE, 2008;14(1),1-23.

[17] Guedes S, Mutti C. Affections in learning situations: a study of an entrepreneurship skills development course. Journal of Workplace Learning. 2010.

[18] Antonacapoulou E, Gabriel J. Emotion, learning and organizational change: Towards an integration of psychoanalytic and other perspectives. Journal of Organizational Change Management. 2001.

[19] Berg C, Hertzog E. Developmental Psychology. 2008.

[20] Fard A, Prusak A. Telling identities: In search of an analytic tool for investigating learning as a culturally shaped activity. Educational Researcher. 2005;34 